\title{
Facet-breaking for three-dimensional crystals evolving by mean curvature
}

\author{
G. BELlETtini \\ Dipartimento di Matematica, Università di Roma "Tor Vergata" \\ via della Ricerca Scientifica, 00133 Roma, Italy. \\ Email:belletti@axp.mat.uniroma2.it \\ M. NovagA \\ Dipartimento di Matematica, Università di Pisa \\ via Buonarroti 2, 56127 Pisa, Italy.Email:novaga@cibs.sns.it
}

AND

M. PAOLINI

Dipartimento di Matematica, Università Cattolica del Sacro Cuore via Trieste 17, 25121 Brescia, Italy.Email:paolini@galileo.dmf.bs.unicatt.it

[Received 6 October 1998]

\begin{abstract}
We show two examples of facet-breaking for three-dimensional polyhedral surfaces evolving by crystalline mean curvature. The analysis shows that creation of new facets during the evolution is a common phenomenon. The first example is completely rigorous, and the evolution after the subdivision of one facet is explicitly computed for short times. Moreover, the constructed evolution is unique among the crystalline flows with the given initial datum. The second example suggests that curved portions of the boundary may appear even starting from a polyhedral set close to the Wulff shape.
\end{abstract}

\section{Introduction}

Motion by crystalline curvature is an anisotropic evolution of a set when the ambient space $\mathbb{R}^{N}$ is endowed with a convex one-homogeneous function whose unit ball (usually called the Wulff shape) is a polyhedron. It provides a geometric model for physical phenomena in phase transitions and crystal growth where the velocity of the evolving front depends on the orientation of the normal vector and has a finite number of preferred directions, corresponding to the facets of the Wulff shape. We refer for instance to [7] for an overview of this kind of geometric evolutions, and to $[3,16,24,25]$ and references therein for some applications. From the mathematical point of view, the presence of corners and flat regions in the Wulff shape is source of interesting problems, as pointed out in the pioneering papers of Taylor [20,21,23] who was also able to compute explicitly the velocity (i.e. the crystalline mean curvature) of the interface (see (4.2)).

The variational nature of this evolution is of basic importance. There is indeed an associated natural free energy, which decreases as fast as possible under crystalline motion by mean curvature, see $[1,2,23]$ and Section 3 below. The simplest situation of motion by crystalline curvature in $N=2$ dimensions has been the object of several recent papers, see for instance $[1,10,11,12,14,18,19]$. In this case, short-time existence and uniqueness of the evolution has been established, as well as an inclusion principle between the evolving fronts. Moreover, if the initial set $E$ is a polygon 
compatible with the Wulff shape, its edges translate parallel to themselves during the evolution, so that no edge-breaking occurs. It is interesting to observe that the situation becomes quite different in presence of a space-dependent forcing term [11]. In the present paper we consider motion by crystalline curvature in $N=3$ dimensions: here the situation is much more complicated, and less is known about the qualitative behaviour of the evolving interfaces. In particular, to the best knowledge of the authors, given an initial set, we can neither definitely predict instantaneous facet-breaking nor understand where subdivisions, if any, take place. In this direction, we refer to the papers $[17,22]$ for numerical simulations. Furthermore, the issue of short-time existence of a 'smooth' evolution is still open, as well as the construction of a unique weak solution [11] describing the motion after the onset of singularities.

A comparison principle has been established in [13] for some particular evolutions in three dimensions, and generalized in any space dimension and for any convex Wulff shape in [4]. This result implies uniqueness of the evolution. We notice that in [13] the study of the crystalline evolution has been carried out under the assumption that no facet-breaking occurs. Here we compute two examples of facet-breaking for three-dimensional polyhedral surfaces evolving by crystalline mean curvature. Example 1 concerns a cubic Wulff shape $\mathcal{W}_{\phi}$ and a non convex $\phi$-regular initial set $E$. It turns out that, for proper choices of the lengths of the edges of $\partial E$, a facet instantly subdivides into two new facets. We explicitly compute the subsequent evolution $t \rightarrow E(t)$ for short times: this evolution is unique, i.e. is the crystalline evolution of $E$. Example 2 is more surprising, even if not completely rigorous: here the Wulff shape is a regular orthogonal prism with hexagonal basis, and the initial set $E$ is a convex polyhedron very close to $\mathcal{W}_{\phi}$. Depending on a certain parameter, there is evidence that a curved portion of the boundary instantly develops. Numerical simulations of all these phenomena will appear in [17]. Such an evolution is expected to be the crystalline evolution of $E$.

These two examples show several facts. Firstly, subdivision of one facet into new facets seems to be a common phenomenon in three dimensions, as already remarked by Taylor in [22] and by Yunger in [26]. Secondly, since it is natural to look for a short time existence theorem in a suitable class of surfaces, then (if our interpretation of Example 2 is correct) this class must be sufficiently large to include piecewise $C^{1}$ surfaces. Notice also that the class of $\phi$-regular polyhedra is not stable under crystalline mean curvature flow, even for short times.

We point out that Taylor expects the existence of initial data for which the amount of new subdivisions is not bounded $[1,21]$. This could be related to our examples.

The plan of the paper is the following. In Section 2 we define some notation. In Section 3 we recall, following [4], the general definitions of $\phi$-regular set and $\phi$-regular flow. These definitions concern basically Lipschitz surfaces; we do not restrict ourselves to polyhedral surfaces, in view of Example 2. At the end of Section 3 we partially compute the first variation of the crystalline perimeter. In Section 4 we give some preliminaries on crystalline evolutions of polyhedral sets. In particular, in Definition 4.1 we introduce the class of $\phi$-calibrable sets. A $\phi$-calibrable set is, roughly speaking, a $\phi$-regular polyhedron with each facet of constant $\phi$-mean curvature, i.e. which admits a global Cahn-Hoffman vector field having constant divergence on each facet. Theorem 4.3 provides a necessary condition for a set to be $\phi$-calibrable, and this will be crucial to construct the examples, which are illustrated in Section 5. We conclude by observing that finding necessary and sufficient conditions on a set $E$ to be $\phi$-calibrable would be very useful to understand the class of initial polyhedral surfaces which do not develop new facets for short times. 


\section{Some notation}

In the following we denote by $\cdot$ the standard euclidean scalar product in $\mathbb{R}^{3}$ and by $|\cdot|$ the euclidean norm of $\mathbb{R}^{3}$. Given a subset $A$ of $\mathbb{R}$ or $\mathbb{R}^{2}$, we denote by $|A|$ the Lebesgue measure of $A$. $\mathcal{H}^{k}$, for $k=1,2,3$, will denote the $k$-dimensional Hausdorff measure in $\mathbb{R}^{3}$. If $E \subset \mathbb{R}^{3}$, we denote by $\partial E$ the topological boundary of $E$. By a polyhedral set $E$ we always mean a bounded closed polyhedral set, and by a facet $F$ (resp. an edge $l$ ) of $\partial E$ (i.e. of $E$ ) we always mean a closed facet (resp. a closed edge); we denote by $\operatorname{int}(F)(\operatorname{resp} . \operatorname{int}(l))$ the relative interior of $F$ (resp. of $l$ ). We assume that all polyhedral sets have a finite number of facets.

Given a polyhedral set $E$, a facet $F$ of $E$ and an edge $l$ of $F$, we denote by $v_{F, l}$ the euclidean unit normal to int $(l)$ lying in the plane of $F$ and pointing outside $F$.

We indicate by $\phi: \mathbb{R}^{3} \rightarrow[0,+\infty[$ a convex function satisfying the properties

$$
\phi(\xi) \geqslant \Lambda|\xi|, \quad \phi(a \xi)=a \phi(\xi), \quad \xi \in \mathbb{R}^{3}, a \geqslant 0,
$$

for a suitable constant $\Lambda \in] 0,+\infty\left[\right.$, and by $\phi^{o}: \mathbb{R}^{3} \rightarrow\left[0,+\infty\left[, \phi^{o}\left(\xi^{*}\right):=\sup \left\{\xi^{*} \cdot \xi: \phi(\xi) \leqslant 1\right\}\right.\right.$ the dual of $\phi$. We set

$$
\mathcal{F}_{\phi}:=\left\{\xi^{*} \in \mathbb{R}^{3}: \phi^{o}\left(\xi^{*}\right) \leqslant 1\right\}, \quad \mathcal{W}_{\phi}:=\left\{\xi \in \mathbb{R}^{3}: \phi(\xi) \leqslant 1\right\} .
$$

$\mathcal{F}_{\phi}$ and $\mathcal{W}_{\phi}$ are convex sets whose interior parts contain the origin. In this paper we shall assume that $\phi$ is crystalline, i.e. $\mathcal{W}_{\phi}$ (and hence $\mathcal{F}_{\phi}$ ) is a convex polytope. $\mathcal{F}_{\phi}$ is usually called the Frank diagram and $\mathcal{W}_{\phi}$ the Wulff shape.

Let $T^{o}: \mathbb{R}^{3} \rightarrow \mathcal{P}\left(\mathbb{R}^{3}\right)$ be the duality mapping defined by

$$
T^{o}\left(\xi^{*}\right):=\frac{1}{2} \partial^{-}\left(\phi^{o}\left(\xi^{*}\right)\right)^{2}, \quad \xi^{*} \in \mathbb{R}^{3},
$$

where $\mathcal{P}\left(\mathbb{R}^{3}\right)$ is the class of all subsets of $\mathbb{R}^{3}$ and $\partial^{-}$denotes the subdifferential in the sense of convex analysis. We observe that $T^{o}$ is a multivalued maximal monotone operator; moreover

$$
T^{o}\left(a \xi^{*}\right)=a T^{o}\left(\xi^{*}\right), \quad a \geqslant 0,
$$

and $T^{o}$ takes $\partial \mathcal{F}_{\phi}$ onto $\partial \mathcal{W}_{\phi}$.

One can show that

$$
\xi^{*} \cdot \xi=\phi^{o}\left(\xi^{*}\right)^{2}=\phi(\xi)^{2}, \quad \xi^{*} \in \mathbb{R}^{3}, \xi \in T^{o}\left(\xi^{*}\right) .
$$

Given a nonempty set $E \subset \mathbb{R}^{3}$ and $x \in \mathbb{R}^{3}$, we set

$$
\begin{gathered}
\operatorname{dist}_{\phi}(x, E):=\inf _{y \in E} \phi(x-y), \quad \operatorname{dist}_{\phi}(E, x):=\inf _{y \in E} \phi(y-x), \\
d_{\phi}^{E}(x):=\operatorname{dist}_{\phi}(x, E)-\operatorname{dist}_{\phi}\left(\mathbb{R}^{3} \backslash E, x\right) .
\end{gathered}
$$

At each point $x$ where $d_{\phi}^{E}$ is differentiable, there holds $\nabla d_{\phi}^{E}(x) \in \partial \mathcal{F}_{\phi}$, i.e. the following eikonal equation holds:

$$
\phi^{o}\left(\nabla d_{\phi}^{E}\right)=1 .
$$


Let $E$ be a polyhedral set and $F$ a facet of $\partial E$. One can check that the function $d_{\phi}^{E}$ is differentiable on $\operatorname{int}(F)$ and we set, for $x \in \operatorname{int}(F)$,

$$
\widetilde{W}_{\phi}^{F}:=T^{o}\left(\nabla d_{\phi}^{E}(x)\right) \subset \partial \mathcal{W}_{\phi}
$$

Notice that $\widetilde{W}_{\phi}^{F}$ is a convex set independent of $x \in \operatorname{int}(F)$. Moreover $\widetilde{W}_{\phi}^{F}$ is a facet of $\partial \mathcal{W}_{\phi}$ if and only if $F$ is a facet of $\partial E$ parallel to a facet of $\partial \mathcal{W}_{\phi}$ (i.e. $\widetilde{W}_{\phi}^{F}$ ) and having the same exterior unit normal.

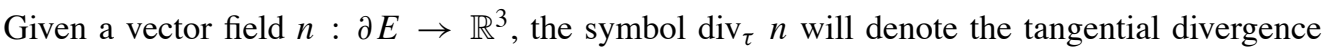
of $n$ on $\partial E$ in the sense of distributions. Finally, the crystalline perimeter is the integral functional defined by

$$
P_{\phi}(E):=\int_{\partial E} \phi^{o}(v) d \mathcal{H}^{2}
$$

where $E$ is a set of finite perimeter and $v$ denotes the outward euclidean unit normal to $\partial E$ (both $\partial E$ and $v$ here must be intended in a geometric measure sense, see [15]). The crystalline perimeter is the natural free energy associated with crystalline motion by mean curvature, see for instance [23], [13] and Section 3 below.

\section{General definitions of $\phi$-regular set and $\phi$-regular flow}

The next definition generalizes to the three dimensional crystalline case the notion of smooth compact surface, compare [4].

Definition 3.1 Let $E \subset \mathbb{R}^{3}$ and $n_{\phi}: \partial E \rightarrow \mathbb{R}^{3}$ be Borel-measurable. We say that the pair $\left(E, n_{\phi}\right)$ is $\phi$-regular if

1. the set $\partial E$ is compact and Lipschitz continuous;

2.

$$
n_{\phi}(x) \in T^{o}\left(\frac{v(x)}{\phi^{o}(v(x))}\right) \quad \mathcal{H}^{2} \text {-a.e. } x \in \partial E
$$

3. there is an open set $A \supseteq \partial E$ such that, for a.e. $y \in A$ there exists a unique $(x, s) \in \partial E \times \mathbb{R}$ so that $y=x+s n_{\phi}(x)$ and, letting $n_{\phi}^{e}(y):=n_{\phi}(x)$, there holds

$$
n_{\phi}^{e} \in L^{\infty}\left(A ; \mathbb{R}^{3}\right), \quad \operatorname{div} n_{\phi}^{e} \in L^{\infty}(A)
$$

4. $\operatorname{div} n_{\phi}^{e}$ admits a trace on $\partial E$, which we denote by $\operatorname{div}_{\tau} n_{\phi} \in L^{\infty}(\partial E)$. Expressed precisely, for $\mathcal{H}^{2}$-a.e., $x \in \partial E$, there exists $\lim _{\rho \rightarrow 0^{+}}\left|B_{\rho}(x)\right|^{-1} \int_{B_{\rho}(x)} \operatorname{div} n_{\phi}^{e}(y) d y=\operatorname{div}_{\tau} n_{\phi}(x)$.

As already remarked in the Introduction, such a generality (as in Definition 3.4 below) is needed in view of Example 2 of Section 5, and could be helpful for a short time existence theorem of $\phi$-regular flows.

If $\partial E$ is a plane, then $\operatorname{div}_{\tau}$ is the usual tangential divergence in the sense of distributions. 
The vector field $n_{\phi}$ is called the Cahn-Hoffman field $[8,9]$; in the case of smooth strictly convex anisotropies $\phi$ and smooth sets $E, n_{\phi}$ is simply given by $T^{o}\left(\nabla d_{\phi}^{E}\right)$.

Definition 3.1 slightly differs from Definition 2.1 of [4], where the vector field $n_{\phi}$ is directly defined in a tubular neighbourhood of $\partial E$ and satisfies $n_{\phi} \in T^{o}\left(\nabla d_{\phi}^{E}\right)$. We anyway expect that, for a large class of functions $\phi$ and of $\phi$-regular sets, the definitions coincide (see Remark 5.5 below). Notice that in the case of smooth anisotropies $\phi$ and smooth sets $E, n_{\phi}$ is extended in a natural way out of the front, and one can check that $\operatorname{div}_{\tau} n_{\phi}=\operatorname{div} n_{\phi}$ on $\partial E$. This essentially follows from the relation $\phi\left(n_{\phi}\right)=1$.

REMARK 3.2 Let $E$ be a polyhedral set having the following property: given any vertex $v$ of $\partial E$, the intersection of $\widetilde{W}_{\phi}^{Q}$ over all facets $Q$ containing $v$ is non empty. Then it is not difficult to prove that there exists a vector field $n_{\phi} \in \operatorname{Lip}\left(\partial E ; \mathbb{R}^{3}\right)$ such that $\left(E, n_{\phi}\right)$ becomes $\phi$-regular.

Concerning the next definition of intrinsic $\phi$-mean curvature we refer to $[6,9,23]$.

Definition 3.3 Let $\left(E, n_{\phi}\right)$ be $\phi$-regular. We define the $\phi$-mean curvature $\kappa_{\phi}$ of $\partial E$ at $\mathcal{H}^{2}$-almost every $x \in \partial E$ as

$$
\kappa_{\phi}:=\operatorname{div}_{\tau} n_{\phi} .
$$

We now introduce the evolution by crystalline mean curvature.

Let $t \in[0, T] \rightarrow E(t) \subset \mathbb{R}^{3}$ be a parametrized family of subsets of $\mathbb{R}^{3}$. Define

$$
d_{\phi}^{E(t)}(x):=\operatorname{dist}_{\phi}(x, E(t))-\operatorname{dist}_{\phi}\left(\mathbb{R}^{3} \backslash E(t), x\right) .
$$

Whenever no confusion is possible, we set $d_{\phi}(x, t):=d_{\phi}^{E(t)}(x)$. Let $T>0$ be given.

Definition 3.4 A $\phi$-regular flow on the interval $[0, T]$ is a family of pairs $\left(E(t), n_{\phi}(\cdot, t)\right)_{t \in[0, T]}$, with $n_{\phi}(\cdot, t): \partial E(t) \rightarrow \mathbb{R}^{3}$, which satisfies the following properties:

(1) $\left(E(t), n_{\phi}(\cdot, t)\right)$ is $\phi$-regular for any $t \in[0, T]$;

(2) the function $d_{\phi}$ is Lipschitz continuous on $\mathbb{R}^{3} \times[0, T]$, differentiable for a.e. $t \in[0, T]$ and for $\mathcal{H}^{2}$-a.e. $x \in \partial E(t)$, and such that

$$
\frac{\partial d_{\phi}}{\partial t}(x, t)=\kappa_{\phi}(x, t), \quad \text { a.e. } t \in[0, T], \mathcal{H}^{2} \text {-a.e. } x \in \partial E(t) .
$$

In [4] a $\phi$-regular flow is defined in a slightly different manner. Essentially (2) is replaced by

$(2) \prime d_{\phi} \in \operatorname{Lip}\left(\mathbb{R}^{3} \times[0, T]\right)$ and

$$
\frac{\partial d_{\phi}}{\partial t}(x, t)=\operatorname{div} n_{\phi}(x, t)+O\left(d_{\phi}(x, t)\right) \quad \text { a.e. }(x, t) \in A \times[0, T],
$$

where $A$ is a suitable tubular neighbourhood of $\partial E$.

As a consequence of Corollary 3.4 in [4], it follows that a $\phi$-regular flow in the sense of [4] depends only on $E(0)$, i.e. it does not depend on the choice of $n_{\phi}$. We obviously expect that in most cases the two notions coincide. Reasoning as in [4], this would imply that two $\phi$-regular flows 
starting from the same set coincide, and also that a comparison principle holds. In any case the two definitions coincide for the evolution of Example 1 (see Remark 5.5), and this shows that the evolution given in Fig. 4 of the initial set $E$ of Fig. 1 is unique.

Finally, in the polyhedral case and if no new facet creates, the evolution law of Definition 3.4 concides with the one considered in [13,23].

We conclude this section by sketching the computation of the first variation of the crystalline perimeter (2.2), which motivates, from a variational point of view, the geometric evolution law in Definition 3.4. The computation of the first variation on all $\phi$-regular sets and for deformations with a generic initial velocity is of course difficult, because of the nondifferentiability of both the surface and the integrand; see $[13,23]$ for some discussion in this direction. We will therefore assume further regularity properties on $\left(E, n_{\phi}\right)$ and on the deformation $\alpha$.

Let $\left(E, n_{\phi}\right)$ be $\phi$-regular and assume that $n_{\phi} \in \operatorname{Lip}\left(A ; \mathbb{R}^{3}\right)$. Let $\alpha \in \operatorname{Lip}\left(A \times \mathbb{R} ; \mathbb{R}^{3}\right)$, with $\alpha(x, s):=x+s g(x)$, for a given $g \in \operatorname{Lip}\left(A ; \mathbb{R}^{3}\right)$ which is assumed to be $\mathcal{H}^{2}$-a.e. differentiable on $\partial E$. Set $\alpha_{s}(x):=\alpha(s, x)$ and $\partial E_{s}:=\alpha_{s}(\partial E)$. Notice that $\alpha_{s}: A \rightarrow \mathbb{R}^{3}$ is bilipschitz for any $s \in\left[-s_{0}, s_{0}\right]$, for $s_{0}$ sufficiently small. Denote by $\mathcal{P}_{\phi}$ the measure $\phi^{o}(v) \mathcal{H}^{2}$ supported on $\partial E$ and set

$$
\begin{aligned}
& v_{\phi}:=\nabla d_{\phi}^{E} \quad \text { a.e. in } A, \\
& X:=\left\{\xi \in \operatorname{Lip}\left(A ; \mathbb{R}^{3}\right), \xi \in T^{o}\left(v_{\phi}\right) \text { a.e. in } A\right\} .
\end{aligned}
$$

Notice that $X$ is non-empty, since $n_{\phi} \in X$, and every $\xi \in X$ admits divergence on $\partial E$. Then the following holds:

$$
\liminf _{s \rightarrow 0} \frac{P_{\phi}\left(E_{s}\right)-P_{\phi}(E)}{s} \geqslant \int_{\partial E} g \cdot v_{\phi} \operatorname{div} \xi d \mathcal{P}_{\phi}, \quad \xi \in X .
$$

The above inequality can be interpreted as follows: the 'subdifferential' of the functional $P_{\phi}$ at $E$ along the field $g$ contains the convex set

$$
D(E):=\left\{\operatorname{div} \xi v_{\phi}: \xi \in X\right\} .
$$

Let us prove (3.3). Denote by $v_{s}$ the outward euclidean unit normal to $\partial E_{s}$. We have

$$
P_{\phi}\left(E_{S}\right)-P_{\phi}(E)=\int_{\partial E} \phi^{o}\left(v_{s}\right)-\phi^{o}(v) \mathrm{d} \mathcal{H}^{2}+s \int_{\partial E} \phi^{o}(v) \operatorname{div}_{\tau} g d \mathcal{H}^{2}+o(s) .
$$

Using the regularity assumptions on $\partial E$ and $g$, and the convexity of $\phi^{o}$, one can check that for $\mathcal{H}^{2}$-a.e. $x \in \partial E$ and for any $\xi \in X$, there holds

$$
\liminf _{s \rightarrow 0} \phi^{o}\left(v_{s}(x)\right)-\phi^{o}(v(x)) \geqslant \xi \cdot \frac{\mathrm{d}}{\mathrm{d} s} v_{s \mid s=0} .
$$

Following [5], Theorem 5.1, we then get

$$
\begin{aligned}
\liminf _{s \rightarrow 0} \frac{P_{\phi}\left(E_{s}\right)-P_{\phi}(E)}{s} \geqslant & \int_{\partial E} \xi \cdot(-v \nabla g+(v \nabla g \cdot v) v) \mathrm{d} \mathcal{H}^{2} \\
& +\int_{\partial E} \phi^{o}(v) \operatorname{div}_{\tau} g \mathrm{~d} \mathcal{H}^{2} .
\end{aligned}
$$


Observe that $\xi \cdot v_{\phi}=1$ in $A$; therefore

$$
\liminf _{s \rightarrow 0} \frac{P_{\phi}\left(E_{s}\right)-P_{\phi}(E)}{s} \geqslant \int_{\partial E} \operatorname{div} g-v_{\phi} \nabla g \xi \mathrm{d} \mathcal{P}_{\phi} .
$$

Decomposing $g$ as $g=\left\langle g, v_{\phi}\right\rangle \xi+\left(g-\left\langle g, v_{\phi}\right\rangle \xi\right)$, (3.3) follows reasoning as in [5]. Given now $\omega^{\xi}:=\operatorname{div} \xi v_{\phi} \in D(E)$, we want to find the field $g^{\xi} \in X$ which solves

$$
\min \left\{\int_{\partial E} g \cdot \omega^{\xi} \mathrm{d} \mathcal{P}_{\phi}: g \in X, \int_{\partial E} \phi(g)^{2} \mathrm{~d} \mathcal{P}_{\phi} \leqslant 1\right\} .
$$

A solution to this problem is given by $g^{\xi}=-c^{\xi} \operatorname{div} \xi \xi$, where $1 /\left(c^{\xi}\right)^{2}=\int_{\partial E}(\operatorname{div} \xi)^{2} \mathrm{~d} \mathcal{P}_{\phi}$, and this motivates Definition 3.4.

\section{The polyhedral case. $\phi$-calibrable sets}

Given a $\phi$-regular pair $\left(E, n_{\phi}\right)$, we say that $F \subset \partial E$ is a planar facet of $\partial E$ if, for some $x \in \partial E, F$, if considered as a subset of $T_{x} \partial E \simeq \mathbb{R}^{2}$ (where $T_{x} \partial E$ denotes the tangent plane at $x$ to $\partial E$ defined $\mathcal{H}^{2}$-a.e. on $\partial E$ ), is the closure of a connected component with Lipschitz boundary of $\partial E \cap T_{x} \partial E$. For $\mathcal{H}^{1}$-a.e. $s \in \partial F$, let $v_{F}(s)$ be the unit exterior normal to $\partial F$ lying in $T_{x} \partial E$. By [13], Lemma 9.2, there is a well defined function $c_{\phi, F} \in L^{\infty}(\partial F)$ which is the trace of $n_{\phi} \cdot v_{F}$ on $\partial F$. For any planar facet $F \subset \partial E$, set $\widetilde{W}_{\phi}^{F}:=T^{o}\left(\nabla d_{\phi}^{E}\right)$. We observe that, given a polyhedron $E, F$ a facet of $\partial E, l$ an edge of $F$ and $s \in \operatorname{int}(l)$, if $\nu_{F}(s)$ points outside $E$ then

$$
c_{\phi, F}(s)=\max \left\{n \cdot v_{F}(s): n \in \widetilde{W}_{\phi}^{F}\right\},
$$

and, if $v_{F}(s)$ points inside $E$, then

$$
c_{\phi, F}(s)=\min \left\{n \cdot v_{F}(s): n \in \widetilde{W}_{\phi}^{F}\right\} .
$$

In particular, $c_{\phi, F}(s)$ does not depend on $s \in \operatorname{int}(l)$. So in the following, we will sometimes denote it by $c_{\phi, F, l}$.

Let us define a $\phi$-calibrable facet of a $\phi$-regular set $\left(E, n_{\phi}\right)$.

Definition 4.1 Let $\left(E, n_{\phi}\right)$ be $\phi$-regular and let $F$ be a planar facet of $\partial E$. We say that $F$ is $\phi$-calibrable if there exists a vector field $n_{\phi, F} \in L^{\infty}\left(F ; \mathbb{R}^{3}\right)$, with $\operatorname{div}_{\tau} n_{\phi, F} \in L^{\infty}(F)$, solving the following problem:

$$
\begin{cases}n_{\phi, F} \in \widetilde{W}_{\phi}^{F} & \mathcal{H}^{2} \text {-a.e. on } F \\ \operatorname{div}_{\tau} n_{\phi, F}=v_{F} & \mathcal{H}^{2} \text {-a.e. on } F \\ n_{\phi, F} \cdot v_{F}=c_{\phi, F} & \mathcal{H}^{1} \text {-a.e. on } \partial F\end{cases}
$$

where the constant $v_{F}$ is uniquely determined by the Gauss-Green theorem $[13,20]$ i.e.

$$
v_{F}:=|F|^{-1} \int_{\partial F} c_{\phi, F}(s) \mathrm{d} \mathcal{H}^{1} .
$$

We say that $E$ is $\phi$-calibrable if there exists a vector field $n_{\phi} \in L^{\infty}\left(\partial E ; \mathbb{R}^{3}\right)$ with $\operatorname{div}_{\tau} n_{\phi} \in$ $L^{\infty}(\partial E)$, such that the restriction of $n_{\phi}$ to each planar facet $F$ of $\partial E$ solves problem (4.1). 
The constant $v_{F}$ defined in (4.2) coincides with the weighted mean curvature introduced by Taylor in the polyhedral case (compare [20,23]), and $-v_{F} \phi^{o}(v) v$ represents the normal velocity of the facet $F$. One can check that the following property holds.

Lemma 4.2 Let $E$ be a $\phi$-calibrable polyhedral set. Then there exists $n_{\phi}: \partial E \rightarrow \mathbb{R}^{3}$ such that $\left(E, n_{\phi}\right)$ is $\phi$-regular and each facet of $\partial E$ has constant $\phi$-mean curvature.

Proof. See Theorem 9.2 of [13].

An interesting problem is to characterize those sets $E \subseteq \mathbb{R}^{3}$ which admit a vector field $n_{\phi}: \partial E \rightarrow$ $\mathbb{R}^{3}$ such that $\left(E, n_{\phi}\right)$ is $\phi$-regular, with every facet of constant $\phi$-mean curvature. Theorem 4.3 below will provide a necessary condition in order to guarantee the existence of such an $n_{\phi}$, and will be useful to construct the examples of Section 5.

THEOREM 4.3 Let $\left(E, n_{\phi}\right)$ be $\phi$-regular and let $F$ be a $\phi$-calibrable planar facet of $\partial E$. Then the following condition holds:

$$
v_{P}:=|P|^{-1} \int_{\partial P} c_{\phi, P}(s) \mathrm{d} \mathcal{H}^{1} \geqslant v_{F},
$$

for any $P \subseteq F$ with Lipschitz boundary, where

$$
c_{\phi, P}(s):= \begin{cases}c_{\phi, F}(s) & \text { if } s \in \partial P \cap \partial F, \\ \sup \left\{n \cdot v_{P}(s): n \in \widetilde{W}_{\phi}^{F}\right\} & \text { otherwise. }\end{cases}
$$

Proof. From (4.1) we get $\operatorname{div}_{\tau} n_{\phi, F}=v_{F}$ on $F$. If we integrate $\operatorname{div}_{\tau} n_{\phi, F}$ over $P \subseteq F$, using the Gauss-Green theorem, we get

$$
|P| \operatorname{div}_{\tau} n_{\phi, F}=\int_{P} \operatorname{div}_{\tau} n_{\phi, F} \mathrm{~d} x=\int_{\partial P} n_{\phi, F} \cdot v_{P} \mathrm{~d} \mathcal{H}^{1} \leqslant \int_{\partial P} c_{\phi, P} \mathrm{~d} \mathcal{H}^{1},
$$

which implies (4.3).

We expect that condition (4.3) is also sufficient for a facet $F \subset \partial E$ to be $\phi$-calibrable, and this is subject of current research. This would suggest that the subdivision condition for a facet $F$ reads as follows: $F$ instantly subdivides if and only if there does not exist a Cahn-Hoffman vector field with constant divergence on $F$ (and with the correct boundary conditions), which becomes equivalent to say that there exists $P \subset F$ with $v_{P}<v_{F}$.

REMARK 4.4 The definitions and results of Sections 3 and 4 can be extended without modifications in arbitrary space dimensions and for a generic convex one-homogeneous function $\phi$.

\section{The examples}

\section{Example 1}

In this example we fix the Wulff shape to be $\mathcal{W}_{\phi}:=[-1,1]^{3}$.

Let $E$ be the set of Fig. 1. Observe that the set $E$ satisfies the assumptions of Remark 3.2. In Proposition 5.2 below we show that, for suitable choices of the lengths of the edges of $\partial E$, there are 


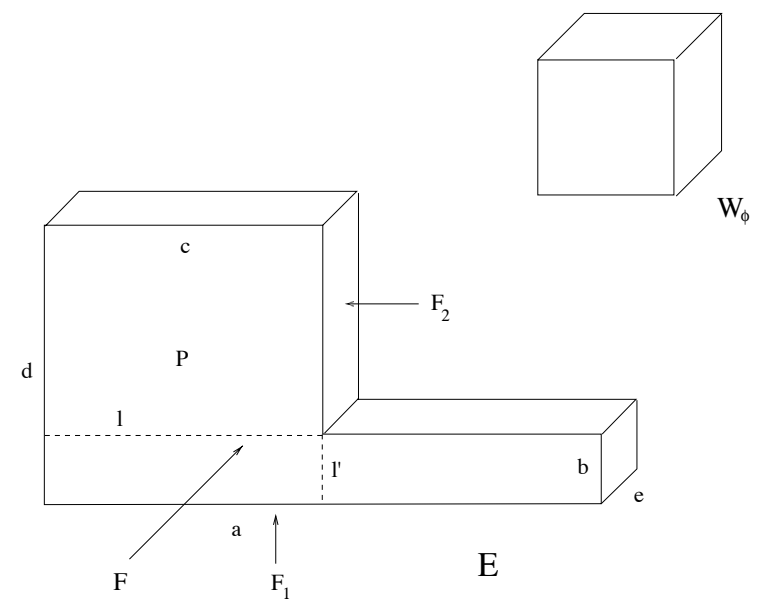

FIG. 1. Example 1: the Wulff shape $\mathcal{W}_{\phi}$ and the initial set $E$.

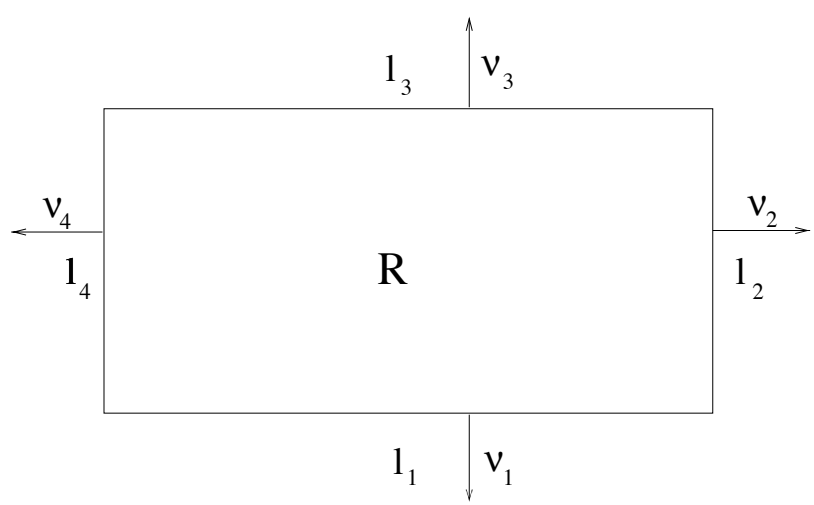

FIG. 2. $\phi$-calibrable rectangular facet.

facets of $\partial E$ not satisfying condition (4.3). In addition, using Lemmas 5.3 and 5.4, we prove that in the crystalline evolution starting from $E$, some facets instantly subdivide. In particular, we observe that the velocity field does not need to be continuous on a facet of the evolving front.

The following lemma shows that a rectangular facet $F$ is always $\phi$-calibrable when $\widetilde{W}_{\phi}^{F}$ is a rectangle; using this fact, we will show that $\phi$-calibrable facets $F$ are not necessarily rectangles.

LEMMA 5.1 Let $R \subset \mathbb{R}^{2}$ be a closed rectangle with edges $l_{1}, \ldots, l_{4}$ parallel to the coordinate axes, and let $v_{i}:=v_{R, l_{i}}$ be the exterior unit normal to $l_{i}$ (see Fig. 2). Let $a_{i}:=a_{l_{i}} \in \mathbb{R}$ $(i=1, \ldots, 4)$ be real numbers with $\left|a_{i}\right| \leqslant 1$. Then there exists a vector field $n=\left(n_{1}, n_{2}\right) \in$ 
$C^{1}\left(\operatorname{int}(R), \mathbb{R}^{2}\right) \cap C\left(R, \mathbb{R}^{2}\right)$ such that

$$
\begin{cases}\max \left\{\left|n_{1}\right|,\left|n_{2}\right|\right\} \leqslant 1 & \operatorname{in} \operatorname{int}(R), \\ \operatorname{div} n=v_{R}:=|R|^{-1} \sum_{i=1}^{4} a_{i}\left|l_{i}\right| & \operatorname{in} \operatorname{int}(R), \\ n \cdot v_{i}=a_{i} & \operatorname{in} \operatorname{int}\left(l_{i}\right) .\end{cases}
$$

Proof. For simplicity, fix the origin at the intersection between $l_{4}$ and $l_{1}$. Given $(x, y) \in R$, we set

$$
n_{1}(x, y):=\frac{a_{2} x}{\left|l_{1}\right|}-a_{4}\left(1-\frac{x}{\left|l_{1}\right|}\right), \quad n_{2}(x, y):=\frac{a_{3} y}{\left|l_{4}\right|}-a_{1}\left(1-\frac{y}{\left|l_{4}\right|}\right) .
$$

The vector field $n:=\left(n_{1}, n_{2}\right)$ satisfies (5.1).

In the following we sometimes identify an edge of a polygon with its length.

Proposition 5.2 Let $F$ be the frontal facet of $E$ with edges of length $a, b, c, d$ respectively (see Fig. 1). Then $F$ is $\phi$-calibrable if and only if

$$
b \geqslant \frac{c d}{c+d}, \quad c \geqslant \frac{a b}{a+b} .
$$

Proof. One directly checks that $c_{\phi, F}=1$ on each edge of $\partial F$, therefore, by the expression of $v_{F}$ in (4.2) we get

$$
v_{F}=\frac{2(a+d)}{c d+b(a-c)} .
$$

Assume now that $F$ is $\phi$-calibrable. Let $P$ be the rectangle in Fig. 1, having edges $c$ and $d$. Recalling the definition of $c_{\phi, P}$ given in Theorem 4.3, we get $c_{\phi, P}=1$ on each edge of $\partial P$. Applying Theorem 4.3 to $P$ and $F$, we get

$$
v_{P}=\frac{2(c+d)}{c d} \geqslant \frac{2(a+d)}{c d+b(a-c)}=v_{F}
$$

i.e.

$$
\frac{c d}{c+d} \leqslant \frac{c d+b(a-c)}{a+d} \text {. }
$$

Rearranging terms, we have

$$
b \geqslant\left(\frac{c d}{c+d}-\frac{c d}{a+d}\right)\left(\frac{a+d}{a-c}\right)=\frac{c d}{c+d},
$$

which is the first inequality of (5.2). The second inequality can be proved in a similar way by applying Theorem 4.3 to $F$ and the rectangle $R$ with edges $a$ and $b$. Indeed, the inequality

$$
v_{R}=\frac{2(a+b)}{a b} \geqslant \frac{2(a+d)}{c d+b(a-c)}=v_{F}
$$

yields

$$
\frac{a b}{a+b} \leqslant \frac{c(d-b)}{a+d}+\frac{a b}{a+d},
$$

i.e.

$$
\left(\frac{a b}{a+b}-\frac{a b}{a+d}\right)\left(\frac{a+d}{d-b}\right) \leqslant c,
$$


which is the second inequality in (5.2).

Assume now that (5.2) holds. We have to prove that $F$ is $\phi$-calibrable. Let us consider the rectangle $F \backslash P$. In order to apply Lemma 5.1 to $F \backslash P$, we have to define the constants $a_{1}, \ldots, a_{4}$. We set these constants equal to 1 on $\partial(F \backslash P) \backslash l^{\prime}$ and we define $a_{l^{\prime}}$ in such a way that

$$
v_{F}=v_{F \backslash P},
$$

i.e.

$$
\frac{1+a_{l^{\prime}}}{2}=\frac{(a+d)(a-c)}{c d+b(a-c)}-\left(\frac{a}{b}-\frac{c}{b}\right)=\frac{(a-c)(c+d-c d / b)}{c d+b(a-c)} .
$$

We need to check that $\left|a_{l^{\prime}}\right| \leqslant 1$. The condition $a_{l^{\prime}} \geqslant-1$ is equivalent to $b \geqslant c d /(c+d)$, whereas the condition $a_{l^{\prime}} \leqslant 1$ is implied by $c \geqslant a b /(a+b)$. Indeed, $\left(1+a_{l^{\prime}}\right) / 2 \leqslant 1$ means that

$$
(d-b) c^{2}-[(d-b)(a+b)+b d] c+a b(d-b) \leqslant 0,
$$

and it is easy to check that the above quadratic polynomial is nonpositive when $c$ satisfies the constraints $a b /(a+b) \leqslant c \leqslant a$. We conclude that (5.2) implies $\left|a_{l^{\prime}}\right| \leqslant 1$.

The same argument applies to the edge $l$. Indeed, for the rectangle $S \subset P$ of edges $c$ and $d-b$, the constant $a_{l}$ is defined in such a way that $v_{F}=v_{S}$, i.e.

$$
\frac{1+a_{l}}{2}=\frac{(d-b)(a+b-a b / c)}{c d+b(a-c)},
$$

and one checks that $\left(1+a_{l}\right) / 2 \in[0,1]$ when $c d /(c+d) \leqslant b \leqslant d$. Once $a_{l}$ and $a_{l^{\prime}}$ have been defined, we can apply Lemma 5.1 three times (separately to the three rectangles partitioning $F$, reversing the signs of $a_{l}$ and $a_{l^{\prime}}$ in the rectangle $P \backslash S$ ) and get a vector field $F \rightarrow \mathbb{R}^{2}$ satisfying (5.1). By adding a constant third component we get a vector field satisfying (4.1).

Notice that the vector field $n_{\phi, F}$ constructed in the last part of the proof of Proposition 5.2 is not continuous on $F$; more precisely the tangential component of $n_{\phi, F}$ along $l^{\prime}$ jumps across $l^{\prime}$.

By choosing for instance $a:=2, b:=\frac{1}{4}, c:=1$, and $d:=1$, it turns out that $F$ is not $\phi$-calibrable, since $v_{F}=\frac{24}{5}, v_{P}=4$, and inequality (4.3) is violated.

We want now to define a $\phi$-regular evolution starting from $E$, when $F$ is not $\phi$-calibrable; notice that, during the evolution, the facet $F$ cannot translate parallel to itself.

We begin with two preliminary lemmas, whose proof is similar to that of Proposition 5.2.

LEMMA 5.3 Let $F_{2}$ be the polygon in Fig. 3. The facet $F_{2}$ is $\phi$-calibrable for any $\left.\epsilon \in\right] 0, e / 2[$ if and only if $b \leqslant e \leqslant d$.

Proof. First we compute the constants $c_{\phi, F_{2}, l}$, where $l$ is an edge of $F_{2}$. We have

$$
c_{\phi, F_{2}, \epsilon}=c_{\phi, F_{2}, d}=c_{\phi, F_{2}, e}=1, \quad c_{\phi, F_{2}, b}=c_{\phi, F_{2}, e-2 \epsilon}=-1,
$$

hence

$$
v_{F_{2}}=\frac{4 \epsilon+2(d-b)}{e(d-b)+2 \epsilon b} .
$$

Assume that $F_{2}$ is $\phi$-calibrable for $\epsilon \in$ ]0,e/2[. Let $Q_{1}$ be the rectangle in Fig. 3, having edges $\epsilon$ and $b$. We have $c_{\phi, Q_{1}, \epsilon}=c_{\phi, F_{2}, \epsilon}=1, c_{\phi, Q_{1}, b}=c_{\phi, F_{2}, b}=-1$, and $c_{\phi, Q_{1}, l^{\prime}}=1$. Hence applying 

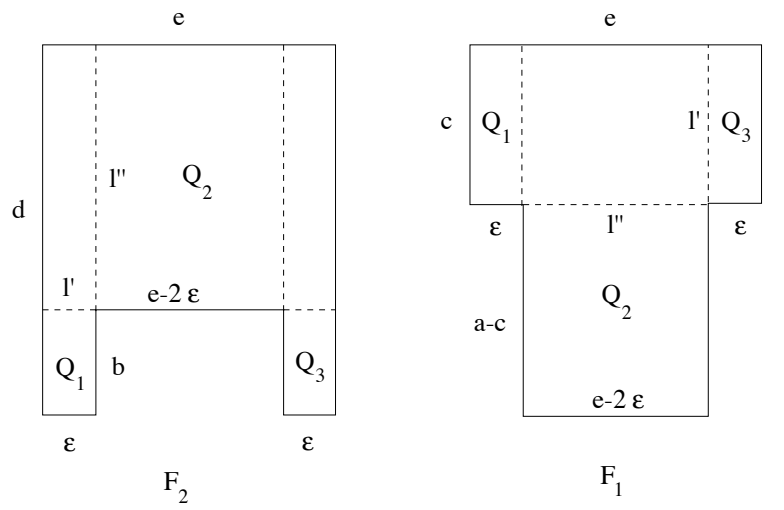

FIG. 3. $\phi$-calibrable facets. The side facet $F_{2}$ and the lower facet $F_{1}$ of $E(t)$.

Theorem 4.3 to $Q_{1}$ and $F_{2}$, we get $v_{Q_{1}}=2 / b \geqslant v_{F_{2}}$, which is equivalent to $b \leqslant e$. The inequality $e \leqslant d$ can be obtained by a similar argument applied to $F_{2}$ and the rectangle $Q_{2}$ of edges $d-b$ and $e-2 \epsilon$ in Fig. 3. Indeed Theorem 4.3 yields

$$
v_{Q_{2}}=\frac{(e-2 \epsilon)+(2 \epsilon-e)+2(d-b)}{(e-2 \epsilon)(d-b)} \geqslant v_{F_{2}},
$$

which is equivalent to $e-d \leqslant 2 \epsilon$. Letting $\epsilon \downarrow 0^{+}$, we get $e \leqslant d$.

Assume now that $b \leqslant e \leqslant d$. We have to show that $F_{2}$ is $\phi$-calibrable for $\left.\epsilon \in\right] 0, e / 2[$. Let us consider the rectangle $Q_{1}$. Choose $a_{l^{\prime}}$ in such a way that $v_{F_{2}}=v_{Q_{1}}$. Since

$$
v_{Q_{1}}=\frac{\epsilon-b+b+\epsilon a_{l^{\prime}}}{\epsilon b}=\frac{1+a_{l^{\prime}}}{b},
$$

we are imposing

$$
\frac{1+a_{l^{\prime}}}{2}=\frac{b(2 \epsilon+d-b)}{e(d-b)+2 \epsilon b} .
$$

Clearly $\left(1+a_{l^{\prime}}\right) / 2 \geqslant 0$, and one directly checks that $b \leqslant e$ is equivalent to $\left(1+a_{l^{\prime}}\right) / 2 \leqslant 1$.

Similarly, let us consider the rectangle $Q_{2}$. We have

$$
v_{Q_{2}}=\frac{2 a_{l^{\prime \prime}}}{e-2 \epsilon} \text {. }
$$

Imposing $v_{F_{2}}=v_{Q_{2}}$ makes the inequality $e-d \leqslant 2 \epsilon$ equivalent to $a_{l^{\prime \prime}} \leqslant 1$, while $a_{l^{\prime \prime}}$ is always $\geqslant$ -1 . Applying Lemma 5.1 and reasoning as in Proposition 5.2, we conclude that $F_{2}$ is $\phi$-calibrable.

Lemma 5.4 Let $F_{1}$ be the polygon in Fig. 3. The facet $F_{1}$ is $\phi$-calibrable for any $\epsilon>0$ sufficiently small if and only if $c \geqslant a e /(a+e)$.

Proof. We have $c_{\phi, F_{1}}=1$ on each edge of $F_{1}$, hence

$$
v_{F_{1}}=\frac{2(a+e)}{a e-2 \epsilon(a-c)} \text {. }
$$


Assume that $F_{1}$ is $\phi$-calibrable and $\epsilon>0$ is sufficiently small. We have $c_{\phi, Q_{3}}=1$ on each edge of $Q_{3}$; hence

$$
v_{Q_{3}}=\frac{2(\epsilon+c)}{\epsilon c} \text {. }
$$

The inequality $v_{Q_{3}} \geqslant v_{F_{1}}$ is always satisfied for $\epsilon$ sufficiently small. Reasoning in a similar way for the rectangle $Q_{2}$, one can check that the inequality

$$
v_{Q_{2}}=\frac{2(a-c)+2(e-2 \epsilon)}{(a-c)(e-2 \epsilon)} \geqslant v_{F_{1}}
$$

is always satisfied for $\epsilon$ sufficiently small.

Assume now that $c \geqslant a e /(a+e)$. We have to prove that $F_{1}$ is $\phi$-calibrable for any $\epsilon>0$ sufficiently small. Choose $a_{l^{\prime}}$ such that $v_{F_{1}}=v_{Q_{3}}$; we have

$$
\frac{1+a_{l^{\prime}}}{2}=\epsilon\left(\frac{(a+e)}{a e-2 \epsilon(a-c)}-\frac{1}{c}\right) .
$$

Hence $\left(1+a_{l^{\prime}}\right) / 2 \leqslant 1$ for $\epsilon$ small enough, and one checks that the inequality $\left(1+a_{l^{\prime}}\right) / 2 \geqslant 0$ is equivalent to

$$
c \geqslant \frac{a e-2 \epsilon(a-c)}{a+e} .
$$

Similarly

$$
v_{Q_{2}}=\frac{2(a-c)+(e-2 \epsilon)\left(1+a_{l^{\prime}}\right)}{(a-c)(e-2 \epsilon)} .
$$

Imposing $v_{F_{1}}=v_{Q_{2}}$, we get

$$
\frac{1+a_{l^{\prime \prime}}}{2}=(a-c)\left(\frac{a+e}{a e-2 \epsilon(a-c)}-\frac{1}{e-2 \epsilon}\right) .
$$

Then $\left(1+a_{l^{\prime \prime}}\right) / 2 \geqslant 0$ whenever $e^{2} \geqslant 4 \epsilon c$, which is satisfied for $\epsilon$ small enough, and one checks that $\left(1+a_{l^{\prime \prime}}\right) / 2 \leqslant 1$ is always satisfied for $\epsilon$ sufficiently small.

Now let $a=2, b=\frac{1}{4}, c=1$, and $d=1$, and choose $e:=\frac{1}{2}$. With this choice of the lengths of the edges of $\partial E$, the facet $F$ is not $\phi$-calibrable, whereas the facets $F_{1}$ and $F_{2}$ are $\phi$-calibrable for $\epsilon$ sufficiently small.

We are in a position to construct an evolution of the set $E$ of Fig. 1, which actually is the evolution of $E$ by crystalline mean curvature; see Remark 5.5. The frontal facet $F$ instantly subdivides into two disjoint facets (the fracture appears along $l^{\prime}$ ), and then each facet translates parallel to itself with velocity given by (4.2). The same applies to the facet opposite to $F$. In this way we get an evolution $t \rightarrow E(t)$ starting from $E$ and defined on a suitable time interval [0,T], with $T>0$, having shape as in Fig. 4. We need to prove that this evolution corresponds to a $\phi$-regular flow starting from $E$. In order to prove this, we must construct a vector field $n_{\phi}(\cdot, t): \partial E(t) \rightarrow \mathbb{R}^{3}$, $t \in[0, T]$, as in Definition 3.4. By Lemma 4.2, it is enough to prove that $E(t)$ is $\phi$-calibrable for $t \in[0, T]$, with $T>0$ sufficiently small. We observe that, for any $t \in[0, T]$, the only facets of $\partial E(t)$ which are not rectangular are $F_{1}$ and $F_{2}$ of Fig. 3. By applying Proposition 5.2 and Lemmas 5.4 and 5.3, it follows that $E(t)$ is $\phi$-calibrable. For $t=0$, one can construct the field $n_{\phi}$ on $\partial E$, by 


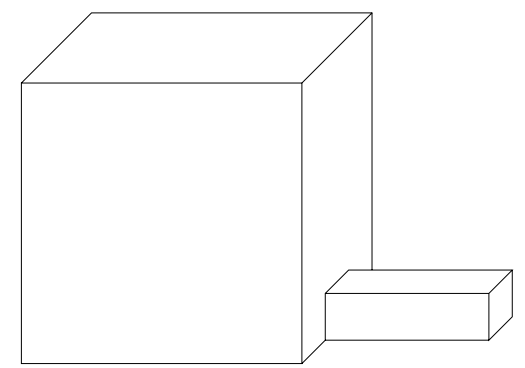

$\mathrm{E}(\mathrm{t})$

FIG. 4. Example 1: qualitative shape of the evolving set $E(t)$ starting from $E$ for short times.

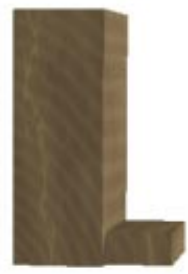

FIG. 5. Example 1: numerical computation of $E(t)$.

considering the facet $F$ (and similarly the facet opposite to $F$ ) as the union of $P$ and $F \backslash P$, and then solve (4.1) independently on the two rectangles, setting $c_{\phi, P, l^{\prime}}=1=-c_{\phi, F \backslash P, l^{\prime}}$. We observe that, with this definition, the field $n_{\phi}$ is not continuous on $F$ : we do not know if there exists a continuous field equivalent to $n_{\phi}$ (i.e. with the same divergence and satisfying the same restrictions).

Figure 5 is the result of a numerical computation.

The following remark is crucial and concerns uniqueness of the $\phi$-regular flow.

REMARK 5.5 Since the vector field $n_{\phi}$ previously defined admits an extension (by lines) in a suitable space-time neighbourhood $A \times[0, T]$ of the evolving front $\partial E(t)$, the map $\left(E(t), n_{\phi}(\cdot, t)\right)$ becomes a $\phi$-regular evolution in the sense of [4], and is unique in that class.

\section{Example 2.}

In this example we fix the Wulff shape to be the regular orthogonal prism with hexagonal basis centred at the origin; the apothem of the hexagonal basis is set equal to 1 . Let $E$ be the convex set of Fig. 6. Observe that also in this case $E$ satisfies the assumptions of Remark 3.2. We will prove that, for a proper choice of the parameter $\epsilon>0, E$ is not $\phi$-calibrable. Moreover, we expect that its evolution develops curve portions of the boundary, i.e. the set $E$ does not remain a polyhedral set under crystalline mean curvature flow. Assume for simplicity that $r=1$ in Fig. 6. 


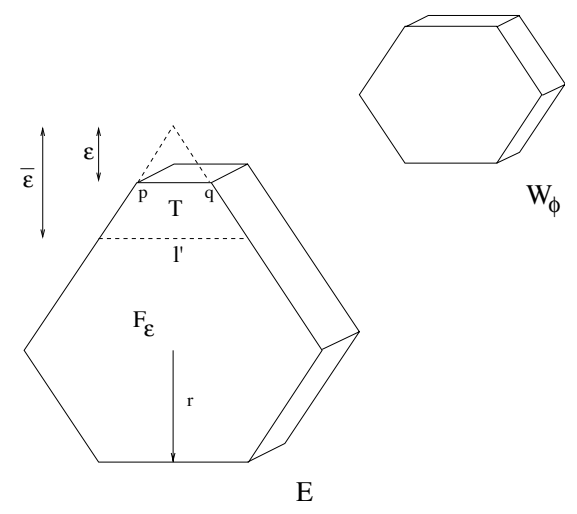

FIG. 6. Example 2: the Wulff shape $\mathcal{W}_{\phi}$ and the initial set $E$.

LEMmA 5.6 There exists $\bar{\epsilon}>0$ such that the frontal facet $F_{\epsilon}$ of $\partial E$ is not $\phi$-calibrable for any $\epsilon \in] 0, \bar{\epsilon}[$.

Proof. We have $c_{\phi, F_{\epsilon}}=1$ on each edge of $\partial F_{\epsilon}$, and

$$
v_{F_{\epsilon}}=\frac{2(7-\epsilon)}{7-\epsilon^{2}} \leqslant 2
$$

for any $\epsilon \in[0,1]$. The function $\epsilon \rightarrow v_{F_{\epsilon}}$ is strictly convex on $[0,1]$, with $v_{F_{0}}=v_{F_{1}}=2$, and attains its minimum for $\bar{\epsilon}=7-\sqrt{42}$, with value $v_{F_{\bar{\epsilon}}}=(7+\sqrt{42}) / 7<2$. Hence $F_{\epsilon}$ is not $\phi$-calibrable for any $\epsilon \in] 0, \bar{\epsilon}[$.

Let us fix $\epsilon \in] 0, \bar{\epsilon}[$. An interesting problem is to understand which is the evolution starting from this set. We expect that such an evolution $E(t)$ does not remain a polyhedral set. This is motivated by the following heuristic argument.

One can realize that $F_{\bar{\epsilon}}$ satisfies (4.3) with the choice $c_{\phi, F_{\bar{\epsilon}}}=1$ on each edge of $\partial F_{\bar{\epsilon}}$. Assuming that condition (4.3) is necessary and sufficient for a facet to be $\phi$-calibrable, we deduce that $F_{\bar{\epsilon}}$ is $\phi$-calibrable. If this is true, there exists a field $n_{\phi}: F_{\bar{\epsilon}} \rightarrow \mathbb{R}^{3}$ satisfying (4.1). Now let $p$ and $q$ be the points in Fig. 6, and let $n(p)$ (resp. $n(q)$ ) be the unique vector obtained as the intersection of $\widetilde{W}_{\phi}^{Q}$ over all facets $Q$ of $\partial E$ having $p$ (resp. $q$ ) as vertex (see Remark 3.2). We define the field $n_{\phi}$ on $T:=F_{\epsilon} \backslash F_{\bar{\epsilon}}$ by taking the linear combination of $n(p)$ and $n(q)$ on every section of $T$ parallel to $l^{\prime}$. We can perform the same construction for the facet of $\partial E$ opposite to $F_{\epsilon}$. Since the other facets of $\partial E$ are all $\phi$-calibrable by Proposition 5.2, we get a global field $n_{\phi}: \partial E \rightarrow \mathbb{R}^{3}$ such that the pair $\left(E, n_{\phi}\right)$ is $\phi$-regular.

We observe that, contrary to Example 1, the function $\operatorname{div}_{\tau} n_{\phi}$ is not constant (nor piecewise constant) on $F_{\epsilon}$, but increases moving away from $l^{\prime}$ in $T$. Moreover, as can be shown by a direct computation, $\operatorname{div}_{\tau} n_{\phi}$ is continuous on $F_{\epsilon}$, i.e. the two definitions of $n_{\phi}$ on $F_{\bar{\epsilon}}$ and on $T$ have the same divergence on $l^{\prime}$. This suggests that, during an evolution starting from $E$, the facet $F_{\epsilon}$ does not break into two different facets as in Example 1, but rather bends inside $E$ (with velocity given by $\operatorname{div}_{\tau} n_{\phi}$ ). Figure 7 (obtained by a numerical computation) shows the expected shape of 


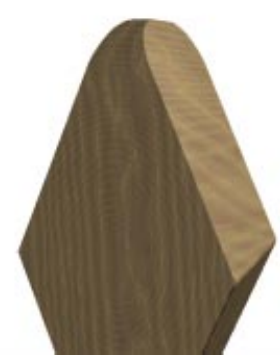

FIG. 7. Example 2: numerical computation of the evolving set $E(t)$ starting from $E$ for short times.

the evolving set $E(t)$ for small positive times. This example suggests that one cannot expect a short-time existence theorem for $\phi$-regular flows in the class of polyhedral sets. The problem of predicting where a fracture creates in a planar facet of an evolving set is interesting and deserves further investigation. Numerical computations in this direction will appear in [17].

\section{Acknowledgements}

This work was partially supported by TMR contract 'Viscosity Solutions and their Applications'.

\section{REFERENCES}

1. Almgren, F. J. \& TAYlor, J. E. Flat flow is motion by crystalline curvature for curves with crystalline energies. J. Diff. Geom. 42, (1995) 1-22.

2. Almgren, F. J., TAYlor, J. E., \& WANG, L. Curvature-driven flows: a variational approach. SIAM J. Control Optimiz. 31, (1993) 387-437.

3. Angenent, S. \& Gurtin, M. E. Multiphase thermomechanics with interfacial structure. II: Evolution of an isothermal interface. Arch. Rational Mech. Anal. 108, (1989) 323-391.

4. Bellettini, G. \& Novaga, M. Approximation and comparison for non-smooth anisotropic motion by mean curvature in $\mathbf{R}^{n}$. Math. Mod. Methods Appl. Sc., to appear.

5. Bellettini, G. \& Paolini, M. Anisotropic motion by mean curvature in the context of Finsler geometry. Hokkaido Math. J. 25, (1996) 537-566.

6. Bellettini, G., Paolini, M., \& Venturini, S. Some results on surface measures in calculus of variations. Ann. Mat. Pura Appl. 170, (1996) 329-359.

7. CAhn, J. W., HANDWERKer, C. A., \& TAYlor, J. E. Geometric models of crystal growth. Acta Metall. Mater. 40, (1992) 1443-1474.

8. CAhn, J. W. \& Hoffman, D. W. A vector thermodynamics for anisotropic interfaces. 1. Fundamentals and applications to plane surface junctions. Surface Sci. 31, (1972) 368-388.

9. CAhn, J. W. \& Hoffman, D. W. A vector thermodynamics for anisotropic interfaces. 2. Curved and faceted surfaces. Acta Metall. Mater. 22, (1974) 1205-1214.

10. Giga, M.-H. \& Giga, Y. Evolving graphs by singular weighted curvature. Arch. Rational Mech. Anal. 141, (1998) 117-198.

11. GigA, M.-H. \& GigA, Y. A subdifferential interpretation of crystalline motion under nonuniform driving force. Dynamical Systems and Differential Equations 1, (1998) 276-287. 
12. Giga, Y. \& Gurtin, M. E. A comparison theorem for crystalline evolutions in the plane. Quarterly of Applied Mathematics 54, (1996) 727-737.

13. Giga, Y., Gurtin, M. E., \& Matias, J. On the dynamics of crystalline motion. Japan Journal of Industrial and Applied Mathematics 15, (1998) 7-50.

14. GiraO, P. M. \& KoHn, R. V. The crystalline algorithm for computing motion by curvature. In SERAPIONI, R. \& TOMARElli, F. (eds), Variational methods for discontinuous structures, pp. 7-18. Birkäuser (1996).

15. GIUSTI, E. Minimal surfaces and functions of bounded variation. Birkhäuser, Boston (1984).

16. GuRTin, M. Thermomechanics of evolving phase boundaries in the plane. Clarendon Press, Oxford (1993).

17. Novaga, M. \& PaOlini, E. A computational approach to fractures in crystal growth. Atti Accad. Naz. Lincei Rend. Ci. Sci. Mat. Fis. Natur. to appear.

18. RoOsEn, A. R. \& TAYLOR, J. Modeling crystal growth in a diffusion field using fully faceted interfaces. J. Comput. Phys. 114, (1994) 444-451.

19. RyBKA, P. A crystalline motion: uniqueness and geometric properties. SIAM J. Appl. Math. 57, (1997) $53-72$.

20. TAYlor, J. E. Crystalline variational problems. Bull. Amer. Math. Soc. (N.S.) 84, (1978) 568-588.

21. TAYLOR, J. E. Constructions and conjectures in crystalline nondifferential geometry. In LAWSON, B. \& Tanenblat, K. (eds), Differential geometry, pp. 321-336. Pitman Monographs in Pure and Applied Math. 52, (1991).

22. TAYLOR, J. E. Geometric crystal growth in 3D via facetted interfaces. In Computational crystal growers workshop, pp. 111-113. Selected Lectures in Mathematics, Amer. Math. Soc. (1992).

23. TAYLOR, J. E. II-mean curvature and weighted mean curvature. Acta Metall. Mater. 40, (1992) 14751485.

24. TILleR, W. A. The science of crystallization. Cambridge University Press (1991).

25. Visintin, A. Models of phase transitions. Birkhäuser, Boston (1996).

26. YUnger, J. Facet stepping and motion by crystalline curvature. $\mathrm{PhD}$ thesis, Rutgers University (1998). 